\title{
Residual Block Error Rate Prediction for IR HARQ Protocol
}

\author{
Mirza HAMZA, Adriana LIPOVAC, Vlatko LIPOVAC
}

\begin{abstract}
This paper provides a simple estimation of the Long Term Evolution (LTE) physical and Medium Access Control (MAC) layer peak transmission performance-the irreducible Block-Error-Rate (BLER) that determines the Hybrid Automatic Repeat Request (HARQ) residual channel available to higher-layer protocols. With this regard, the general pre-HARQ BLER prediction is developed for the redundancy version 0 (RV0) codeword transmission, expressed by the Bit-Error-Rate (BER)), considering the cyclic prefix protection against inter-symbol interference sufficient to prevent long error bursts. This implies only sporadic bit error occurrences, exhibiting moderate mutual interdependence that we modelled considering errored bits of each block of data as a sample without replacement and consequently describing it with the hypergeometric distribution instead of the mostly used binomial one. The HARQ BLER estimation model is verified by both problem-dedicated Monte-Carlo simulations and industry-standard LTE software simulation tool, specifically for the LTE FDD downlink channel environment, as the test results exhibit excellent matching with the residual BLER prediction.
\end{abstract}

Keywords: BER; BLER; HARQ; OFDM

\section{INTRODUCTION}

With evolving standards for digital transmission systems performance from bit-centric to block-centric ones, and deployment of Long Term Evolution (LTE) systems of fourth generation ( $4 \mathrm{G})$, the probability of a bit error-in practice commonly referred to as the Bit-ErrorRate $(B E R)$, has been finally removed from specifications retaining just the Block-Error-Rate $(B L E R)$ as the only transmission performance key indicator [1].

Actually, even a long time ago, it came out that $B E R$ did not solely characterize the transmission system performance, since it could not make distinction between the random - essentially uniform distribution of (mostly sporadic) bit-errors in time, and the bursty one [1,2].

So, although this primarily pertains to the physical layer alone, during the course of time, it came out that instead of bits, it is the (transmission performance of) data blocks that need to be tested. This applies upwards the protocol stack to erroneous protocol data units (PDU) at higher layers as well, indicating their protocols' transmission performance.

Another important request of the LTE transmission performance specifications is the capability of in-service BLER testing by counting the Hybrid Automatic Repeat Request (HARQ) error recovery protocol negative acknowledgements (NACK), relative to their overall count (that includes the positive ones as well). Though in-service performance testing is definitely attractive to network operators that prefer to continue their revenue-generating traffic (rather than interrupt it by out-of-service BER tests), it can compromise accuracy of small $B L E R$ values, if a receiver repeat request, for any reason, fails to reach the transmitter. This can degrade the performance of higher protocols, such as e.g. TCP, which starts to become less tolerant to $B L E R$ larger than $10^{-5}$ ).

Accordingly, the state-of-the-art cross-layer system design involves not only the physical layer performance itself, but also its "spreading" through the upward Medium Access Control (MAC), all the way up to higher protocols, which defines the so-called residual channel, determined by the minimal BLER that the physical layer can deliver [3, 4].
However, though BER has been removed as transmission performance indicator (in favour of $B L E R$ ) from network operator specifications, it has remained inevitable in design, research and development, and manufacturing of network equipment [5-7]. So, in many practical situations, it is useful to quickly estimate $B L E R$ based on given $B E R$ achievable through the residual channel of interest.

Accordingly, in Section 2, we firstly derive the preHARQ general BLER estimation (from known $B E R$ ), considered as related to the redundancy version 0 (RV0) codeword. Then the Incremental Redundancy (IR) HARQ error process is modelled to integrate the general $B L E R$ model into MAC layer LTE environment. In Section 3, simulation test results are presented, while final conclusions are given in Section 4.

\section{RESIDUAL BLER MODEL \\ 2.1 Physical Layer General BLER Model}

General relationship between $B L E R$ and $B E R$ (where the latter jointly and concisely expresses performance degradation due to additive white Gaussian noise (AWGN) and various other impairments), is significantly influenced by time distribution of erroneous bits, ranging from sporadic (with random occurrences mostly due to dominating additive noise), to bursty (e.g. due to multipath propagation).

In this respect, let us start from the simplified model [1], considering $N$-long bit sequence transmitted in $M$ successive blocks.

Suppose that $K$ bit errors overall are spread over $M$ data blocks of length $L$ each, where:

$L=\frac{N}{M}$

The average (long-term) BER is then:

$B E R=\frac{K}{N}=p$

while with $K_{i}$ erroneous bits in the $i$-th block, the corresponding short-term $B E R_{i}$ is: 
$B E R_{i}=\frac{K_{i}}{L}=M \cdot \frac{K_{i}}{N}=p_{i}$

Further on, we consider any block of data with at least a single bit error, to be an Errored Block (EB) [1]. This determines the BLER to be the count of EBs relative to the overall count $M$ of test data blocks. Even for fixed total errors count $K$ (and so the fixed long-term average $B E R=$ $p), B L E R$ is strongly affected by time distribution of errors and by block size $L$. So, with quasi-uniformly timedistributed sporadic bit error occurences, many EBs and consequently frequent HARQ retransmissions occur, which implies that, in this case, shorter blocks maximize data throughput (while for rare retransmissions, longer blocks are preferred with this respect). On the contrary, error bursts leave long error-free intervals, so longer blocks (with reduced relative PDU overhead and increased goodput) can be afforded as EB retransmissions occur rarely. In this regard, assuming sporadic error occurences when their actual distribution is bursty, may result with overestimated $B L E R$, and vice versa [1].

The relationship between $B L E R$ and $B E R$ has been extensively modeled [8]. With respect to this, we adopt that the EB occurences are mutually independent for various reasons, such as supposedly long enough cyclic prefix, interleaving etc.

Moreover, let us adopt that, out of fixed overall count $K$ of bit errors (i.e. for fixed long-term $B E R$ ), the bit errors that already occured in previous blocks do not determine $B E R_{i}$ of the observed (actual) and the following blocks. So, this introduces moderate interdependence among bit and block error occurrences, which can be appropriately addressed by statistical model of sampling without replacement. Consequently, instead of common binomial, we adopt the hypergeometric distribution of probability $P\left(K_{i}\right)$ that any $L$-long EB comprises $K_{i}$ errors [2]:

$P\left(K_{i}\right)=\frac{\left(\begin{array}{l}K \\ K_{i}\end{array}\right)\left(\begin{array}{l}N-K \\ L-K_{i}\end{array}\right)}{\left(\begin{array}{l}N \\ L\end{array}\right)}$

where the natural numbers $N, L$ and $K$ satisfy the condition:

$\max (0, L-N+K) \leq K_{i} \leq \min (L, K)$ block is:

Eq. (4) implies that the probability of an error-free data

$P\left(K_{i}=0\right)=\frac{\left(\begin{array}{c}N-K \\ L\end{array}\right)}{\left(\begin{array}{c}N \\ L\end{array}\right)}=\frac{(N-K) ! \cdot(N-L) !}{N ! \cdot(N-K-L) !}$

whose1-complement, i.e. BLER, comes out of the EB definition:

$B L E R=1-P\left(K_{i}=0\right)=1-\frac{(N-K) ! \cdot(N-L) !}{N ! \cdot(N-K-L) !}$
Now let us simplify Eq. (7) applying the Stirling approximation for factorial function of a large number $n$ [9]:

$n ! \approx n^{n} \cdot e^{-n} \cdot \sqrt{2 \pi n}$

in both numerator and denominator of Eq. (7), so it becomes [2]:

$$
B L E R \approx 1-\frac{\left(1-\frac{K}{N}\right)^{N-K+\frac{1}{2}}}{\left(1-\frac{K}{N-L}\right)^{N-K-L+\frac{1}{2}}} \cdot\left(1-\frac{L}{N}\right)^{K}
$$

Finally, substituting Eq. (2) into Eq. (9) provides the following closed-form for the $B L E R$ vs $B E R=p$ general relationship [2]:

$$
B L E R(p) \approx 1-\frac{(1-p)^{N(1-p)+\frac{1}{2}}}{\left(1-\frac{p \cdot N}{N-L}\right)^{N(1-p)-L+\frac{1}{2}}} \cdot\left(1-\frac{L}{N}\right)^{p \cdot N}
$$

The Eq. (10) can be verified under limit conditions, such as with $p=0$, implying $B L E R=0$, or with any $p>0$ and very long block(s): $L \rightarrow N$ (i.e. $M \rightarrow 1$ ), when $B L E R$ expectedly becomes close to unity.

Furthermore, with many data blocks: $M>>1$, i.e. $L<<$ $N$, and arbitrary BER, Eq. (10) simplifies to [2]:

$$
B L E R_{M>>}(p) \approx 1-\frac{(1-p)^{N(1-p)+\frac{1}{2}}}{(1-p)^{N(1-p)-L+\frac{1}{2}}}=1-(1-p)^{L}
$$

Moreover, with very short blocks whose length almost approaches a single bit: $L \rightarrow 1$, Eq. (11) leads to the expected consequence that: $B L E R \rightarrow B E R$.

From the other side, for extremely small $B E R$ (and moderate block length $L$ ), specifically when:

$$
p<<1-\frac{L}{N}=1-\frac{1}{M}
$$

Eq. (10) reduces to [2]:

$$
\operatorname{BLER}_{p<<}(p) \approx 1-\left(1-\frac{L}{N}\right)^{p \cdot N}
$$

\subsection{HARQ Residual BLER Model}

Having modelled the physical layer block-oriented transmission performance, let us now move upwards the protocol stack and take into account the IR-HARQ blockerror recovery through PHY/MAC layers.

In this regard, without any loss in generality, let us consider the so far handled block to be the redundancy 
version 0 (RV0) out of four IR-HARQ codeword transmissions (RV0 to RV3), presented in Fig. 1 for Modulation Coding Scheme (MCS) index 6, AWGN channel and the bandwidth of 25 Resource Blocks (RB) [10]. As it can be seen from this particular example, the higher RVs reach the target reference $B L E R$ value of $10 \%$ with lesser $S N R$ due to their coding gains $G_{\mathrm{RV} i / 0} ; i=1, \ldots$, 3.

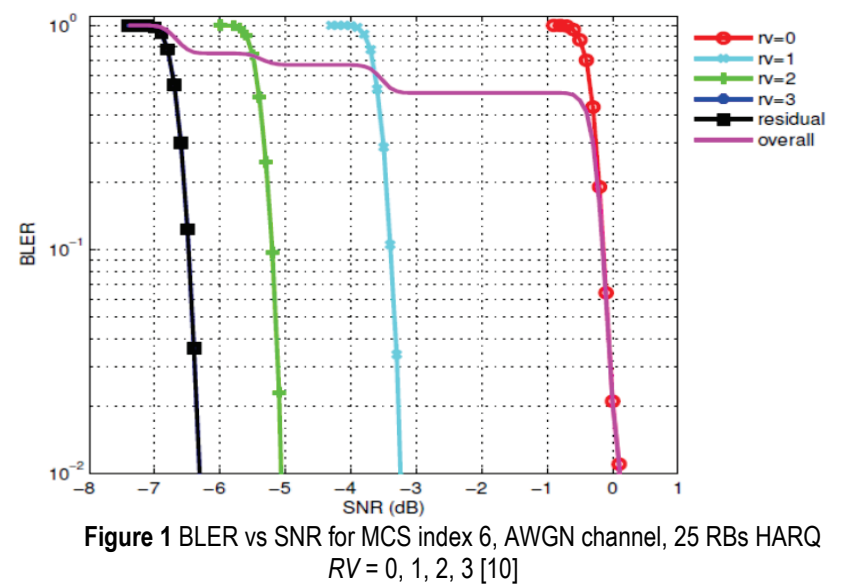

Specifically, the attribute residual relates to the RV3, which in this example, at $B L E R=10 \%$, exhibits the coding gain $G_{\mathrm{RV} 3 / 0} \approx 6.4$ with respect to the RV0, and determines the performance that is delivered to the ultimate ARQ procedure at the Radio Link Control (RLC) layer [11]. (We can easily insert it in our model simply by observing ARQ block as RV4 and taking into account its repetition gain with respect to RV3 as contributing to the overall $G_{\mathrm{RV} 4 / 0}$, which implies the after-ARQ $B L E R$ to be $B L E R_{\mathrm{RES}}=$ $\left.B L E R_{\mathrm{RV} 4}\right)$.

Moreover, the coding gains at the reference $B L E R$ value of $10 \%$, monotonically increase with the MCS index (i.e. with higher modulation formats and code rates) not only absolutely but also relatively in the sense of exhibiting larger dispersion among the RVs [11].

Accordingly, at any chosen "operating point", i.e. particular MCS, i.e. Channel Quality Indicator (CQI) index of interest, the post-HARQ residual $B L E R$, namely: $B L E R_{\mathrm{RES}}=B L E R_{\mathrm{RV} 3}$, can be estimated by applying the gain $G_{\mathrm{RV} 3 / 0}$ of the RV3 with respect to RV0, onto the pre-HARQ $B L E R_{\mathrm{RV} 0}$, where we consider all channel impairments, including not only the AWGN and the similarly behaving co-channel and adjacent channel radio interference, but also non-additive and non-linear ones (e.g. non-linearity of high-power amplifiers, which distorts modulation constellation and produces intersymbol interference (ISI), which finally causes symbol errors) to be abstracted by the equivalent AWGN channel [10].

However, unlike the coding gain $G_{\mathrm{RV} 3 / 0}$ that is easy to read from MCS/CQI-specific BLER graphs, the corresponding $B L E R$ increments are difficult to measure this way between the steep waterfall $B L E R$ curves (such as the ones in Fig. 2), specifically for longer blocks and larger bandwidth (defined by the count of Resource Blocks (RB)) [10].

Therefore, having chosen a certain $\mathrm{MCI} / \mathrm{CQI}$ value and so the modulation type and the coding gain $G_{\mathrm{RV} 3 / 0}$ between the RV0 and the RV3, the residual BLER is defined by:

$$
B L E R_{\mathrm{RES}}\left[\frac{\left(\frac{E_{\mathrm{b}}}{N_{0}}\right)_{\mathrm{RV} 0}}{G_{\mathrm{RV} 3 / 0}}\right]=B L E R_{\mathrm{RV} 0}\left[\left(\frac{E_{\mathrm{b}}}{N_{0}}\right)_{\mathrm{RV} 0}\right]
$$

where we assume that Eq. (14) is not strictly applicable just for the target $B L E R=10 \%$, but for the entire $E_{\mathrm{b}} / N_{0}$ range.

So, now we can apply the $B L E R$ Eq. (10), Eq. (11) or Eq. (13)-let us select the middle one (implying many blocks: $M>>1$ ) into Eq. (14), which makes the post-HARQ residual $B L E R$ :

$$
B L E R_{\mathrm{RES}}=1-\left[1-B E R_{\mathrm{RV} 3}\right]^{L}
$$
[4]:

Specifically, for the RV0 and RV3, the actual BER is

$$
\begin{aligned}
& B E R_{\mathrm{RV} 0}=k_{\mathrm{MOD}} \cdot Q\left(\sqrt{2 \cdot\left(\frac{E_{\mathrm{b}}}{N_{0}}\right)_{\mathrm{RV} 0}}\right) \\
& B E R_{\mathrm{RV} 3}=k_{\mathrm{MOD}} \cdot Q\left[\sqrt{2 \cdot\left(\frac{E_{\mathrm{b}}}{N_{0}}\right)_{\mathrm{RV} 3}}\right]
\end{aligned}
$$

respectively, where $Q(\cdot)$ is a Gaussian tail function, and $k_{\mathrm{MOD}}$ indicates the specific LTE-relevant $m$-QAM modulation format (i.e. with $m$ equal to 4,16 and 64 with Gray constellation mapping), and takes the following values:

$k_{\mathrm{MOD}}=\frac{\frac{S E R_{m \mathrm{QAM}}}{B E R_{\mathrm{BPSK}}}}{1 \mathrm{~d} m}=\left\{\begin{array}{l}2 / 2=1 ; m=4 \text { for } 4-Q A M \\ 3 / 4=0.75 ; m=16 \text { for } 16-Q A M \\ 3.5 / 6=0.583 ; m=64 \text { for } 64-Q A M\end{array}\right.$

where 2, 3 and 3.5 are the average numbers of nearest neighbors in 4-QAM, 16-QAM and 64-QAM constellations, respectively, determining the $S E R_{m \mathrm{QAM}}$ by the BPSK SER, i.e. its BER.from [4].

From (18), we obtain for the RV0:

$\left(\frac{E_{\mathrm{b}}}{N_{0}}\right)_{\mathrm{RV} 0}=\frac{\left[Q^{-1}\left(\frac{B E R_{\mathrm{RV} 0}}{k_{m \mathrm{QAM}}}\right)\right]^{2}}{2}$

Then we apply the coding gain $G_{\mathrm{RV} 3 / 0}$ to Eq. (19) for the RV3:

$\left(\frac{E_{\mathrm{b}}}{N_{0}}\right)_{\mathrm{RV} 3}=G_{\mathrm{RV} 3 / 0} \cdot\left(\frac{E_{\mathrm{b}}}{N_{0}}\right)_{\mathrm{RV} 0}=G_{\mathrm{RV} 3 / 0} \cdot \frac{\left[Q^{-1}\left(\frac{B E R_{\mathrm{RV} 0}}{k_{m \mathrm{QAM}}}\right)\right]^{2}}{2}$

so that Eq. (17) modifies to:

$B E R_{\mathrm{RV} 3}=k_{m \mathrm{QAM}} \cdot Q\left[\sqrt{G_{\mathrm{RV} 3 / 0}} \cdot \sqrt{2 \cdot\left(\frac{E_{\mathrm{b}}}{N_{0}}\right)_{\mathrm{RV} 0}}\right]$ 
Moreover, taking into Eq. (19), $B E R_{\mathrm{RV} 3}$ in Eq. (21) can be expressed as a function of $B E R_{\mathrm{RV} 0}$ :

$$
B E R_{\mathrm{RV} 3}=k_{m \mathrm{QAM}} \cdot Q\left[\sqrt{G_{\mathrm{RV} 3 / 0} \cdot\left[Q^{-1}\left(\frac{B E R_{\mathrm{RV} 0}}{k_{m \mathrm{QAM}}}\right)\right]^{2}}\right]
$$

Furthermore, we can substitute Eq. (22) into Eq. (15) to obtain:

$$
B E R_{\mathrm{RV} 3}=k_{m \mathrm{QAM}} \cdot Q\left[\sqrt{G_{\mathrm{RV} 3 / 0} \cdot\left[Q^{-1}\left(\frac{B E R_{\mathrm{RV} 0}}{k_{m \mathrm{QAM}}}\right)\right]^{2}}\right]
$$

However, for small $B E R_{\mathrm{RV} 3}$ values, we can approximate:

$1-B E R_{\mathrm{RV} 3} \approx \mathrm{e}^{-B E R_{\mathrm{RV} 3}}$

so that, having in mind Eq. (21) and Eq. (24), Eq. (23) simplifies to:

$B L E R_{\mathrm{RES}} \approx 1-\mathrm{e}^{-L \cdot k_{m \mathrm{QAM}} \cdot Q\left[\sqrt{G_{\mathrm{RV} 3 / 0} \cdot\left[Q^{-1}\left(\frac{B E R_{\mathrm{RV} 0}}{k_{m \mathrm{QAM}}}\right)\right]^{2}}\right]}$

Moreover, for very small $B E R_{\mathrm{RV} 3}$, Eq. (24) is brought down to:

$$
B L E R_{\mathrm{RES}} \approx L \cdot B E R_{\mathrm{RV} 3}
$$

while the residual BLER prediction Eq. (25) finally simplifies to:

$$
B L E R_{\mathrm{RES}} \approx L \cdot k_{m \mathrm{QAM}} \cdot Q\left[\sqrt{G_{\mathrm{RV} 3 / 0} \cdot\left[Q^{-1}\left(\frac{B E R_{\mathrm{RV} 0}}{k_{m \mathrm{QAM}}}\right)\right]^{2}}\right]
$$

As it is obvious from Eq. (23), Eq. (25), Eq. (27), larger coding gain $G_{\mathrm{RV} 3 / 0}$ expectedly implies smaller $B L E R_{\mathrm{RES}}$.

In fact, $\sqrt{G_{\mathrm{RV} 3 / 0}}$ can be interpreted as standard deviation of a normal random variable that is integrated by the $Q$-function from $\sqrt{2 \cdot\left(\frac{E_{\mathrm{b}}}{N_{0}}\right)_{\mathrm{RV} 0}} \cdot \sqrt{G_{\mathrm{RV} 3 / 0}}$ on. Likewise, considering $B L E R_{\mathrm{RV} 0}$ as a special case with unity coding gain $G_{\mathrm{RV} 3 / 0}=1$, the standard normal random variable is integrated by the $Q$-function from $\sqrt{2 \cdot\left(\frac{E_{\mathrm{b}}}{N_{0}}\right)_{\mathrm{RV} 0}}$ on.

\section{TEST RESULTS}

3.1 Verification of pre-HARQ Performance by MC Simulations

We validated the developed pre-HARQ model by means of Monte Carlo (MC) simulations, using MATLAB simulation tool. The test bit-sequence pattern and the particular erroneous bits were randomly selected over the blocks so as to follow the hypergeometric distribution Eq. (4).

Accordingly, BLER estimation Eq. (10) and MC simulation results are graphed in Fig. 2, exhibiting excellent matching [2].

Moreover, for applied large $N$, and $L$ taking the LTE maximal value of 6144 bits, the values coming out of specific BLER estimations Eq. (11) and Eq. (13), coincide with the ones resulting from Eq. (10), as it can be seen in Fig. 3.

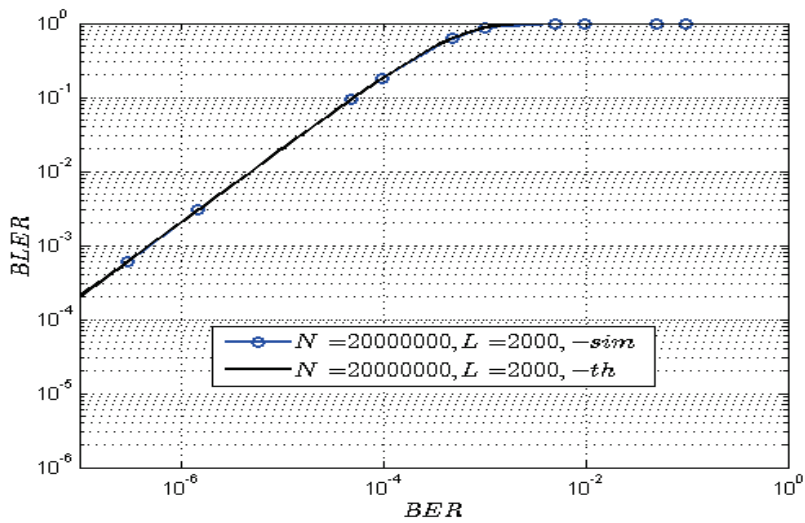

Figure $2 B L E R$ vs $B E R$ ( $N$-long test pattern, $L$-long blocks); predicted (th) vs MC-simulated (sim) [2]

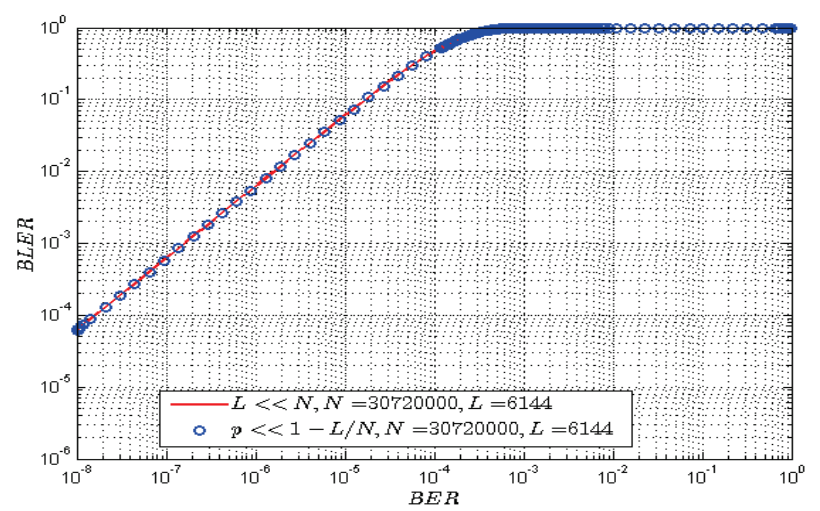

Figure $3 B L E R$ vs $B E R$ (many short blocks: $M>>1, L<<N$, very small $B E R$ : $p<<1-L / N)$

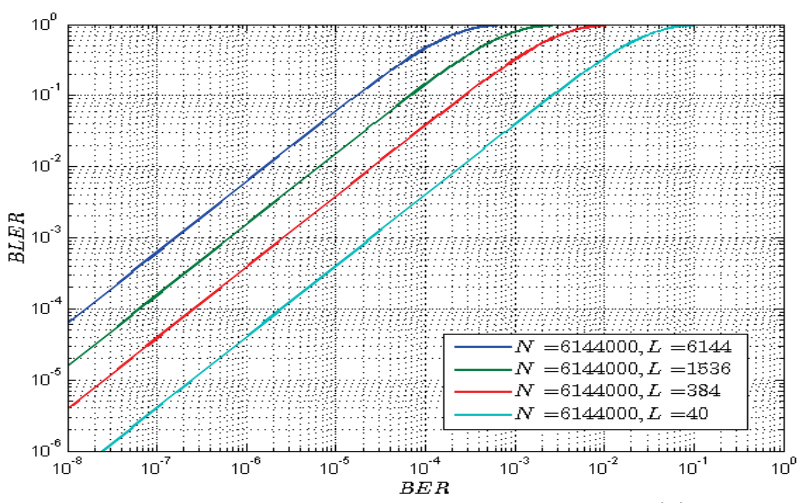

Figure 4 Predicted- $B L E R$ vs $B E R$ dispersion with block length $(L)$; constant test pattern length $(N)$

The predicted-BLERdispersion with block length is evident in Fig. 4, and can be explained by deficit of bit errors at low $B E R$ values to turn many shorter blocks to EBs (which implies lower BLER), while being quite 
sufficient in this regard with not so many large blocks (which implies larger $B L E R$ ).

In addition, we verified the developed $B L E R$ vs $B E R$ expressions by means of the SIMULINK model for the AWGN channel. As it can be seen in Fig. 5, this way obtained $B L E R$ values evidently exhibit very good matching with their corresponding predicted-BLER values, and finally with the ones resulting from adopting hyper geometrically distributed bit errors within the EBs.

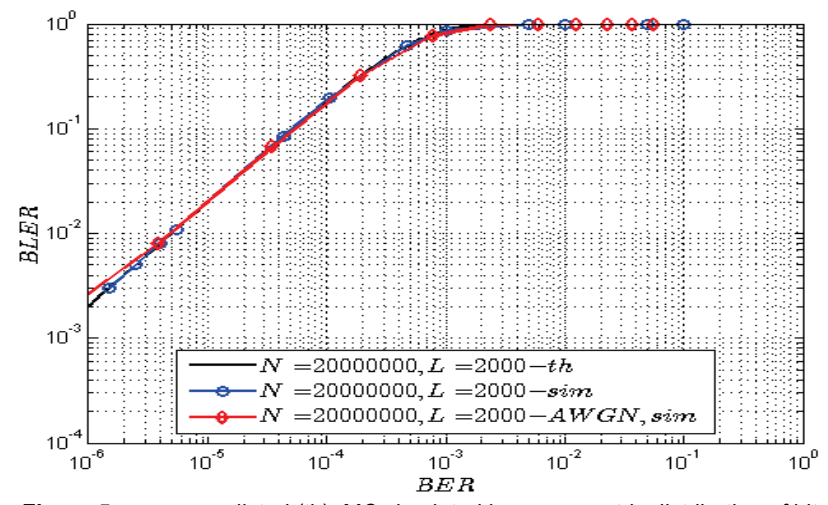

Figure 5 BLER: predicted (th), MC simulated hypergeometric distribution of bit errors (sim), SIMULINK backed simulation (AWGN, sim)

\subsection{Verification of Post-HARQ Performance by MC Simulations}

With small $B E R_{\mathrm{RV} 0}$, Eq. (23), Eq. (25) and Eq. (27) for the post-HARQ residual $B L E R$ exhibit very close values, both mutually as well as with the corresponding dedicated MC simulations.

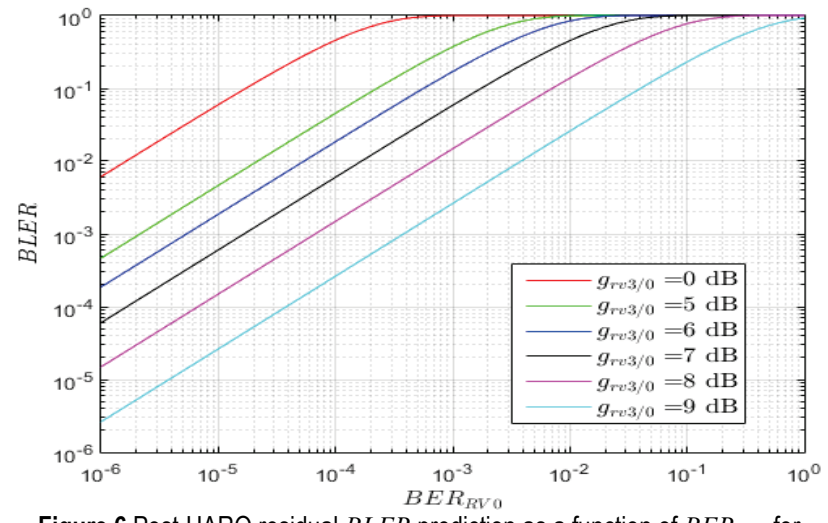

Figure 6 Post-HARQ residual $B L E R$ prediction as a function of $B E R_{\mathrm{RV} 0}$ for various coding gains

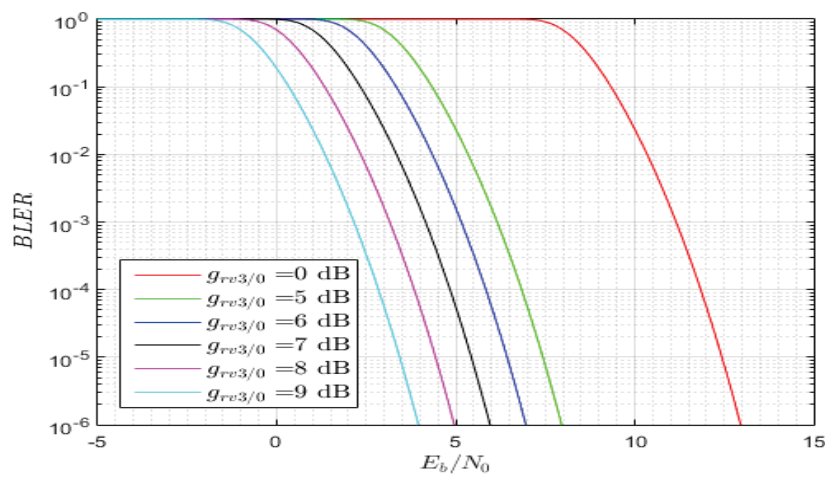

Figure 7 Residual $B L E R$ prediction as a function of $E_{\mathrm{b}} / N_{0}$ for various coding gains
In Fig. 6, $B L E R_{\mathrm{RES}}$ is graphed as a function of $B E R_{\mathrm{RV} 0}$ for various typical IR-HARQ coding gains $G_{\mathrm{RV} 3 / 0}$, where 0 $\mathrm{dB}$ is assigned to the RV0. The corresponding classical $B L E R_{\mathrm{RES}}$ vs $E_{\mathrm{b}} / N_{0}$ curves are presented in Fig. 7 as a reference.

\subsection{Verification Using Standard LTE Simulator}

The derived expression for pre-HARQ $B L E R$ estimation Eq. (10) from known $B E R$, is applicable to any PDU upwards the protocol stack. However, specifically for the post-HARQ $B L E R_{\mathrm{RES}}$ vs $B E R_{\mathrm{RV} 0}$ model validation not only by particular problem oriented MC simulations, we used a more sophisticated and industry-standard test tool-the LTE system-level simulator-SystemVue from Agilent Technologies [7, 13], as an excellent tool for validation of the developed model, specifically in LTE environment, by testing BER and BLER of the 3GPP LTE FDD downlink [2]. The LTE parameters' values that we chose conformed to the ones used in MC simulations.

So, in Fig. 8, the $B L E R$ vs $S N R$ graph for $M C S / C Q I=$ 6 is presented, while the corresponding $B E R$ vs $S N R$ curve is shown in Fig. 9.

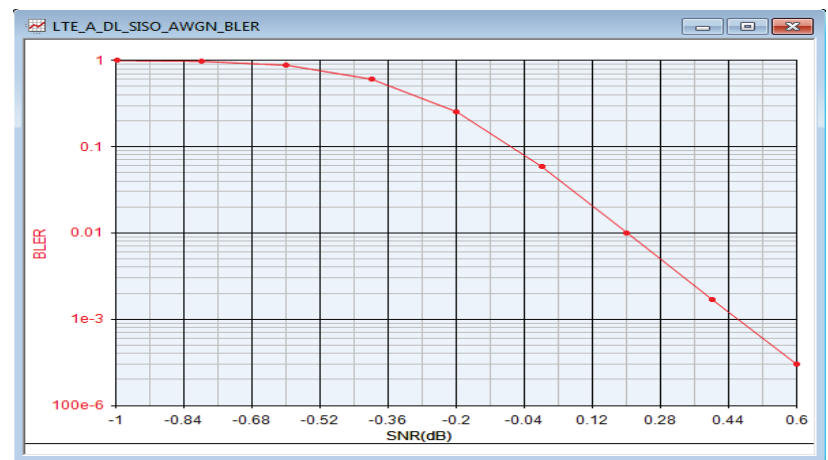

Figure $8 B L E R_{\mathrm{RES}}$ VS $S N R$ (AWGN channel, $M C S / C Q I=6$ )

The BLER and BER graphs presented in Fig. 8 and Fig. 9 , respectively, have quite similar shape, which indicates that the dominant cause of errors is additive noise in the channel (as the long-enough cyclic prefix reliably prevents the ISI and error bursts, leaving just sporadic errors distributed over that many distinct EBs). Therefore, fairly high $B L E R$ values are obtained for not too small $B E R$.

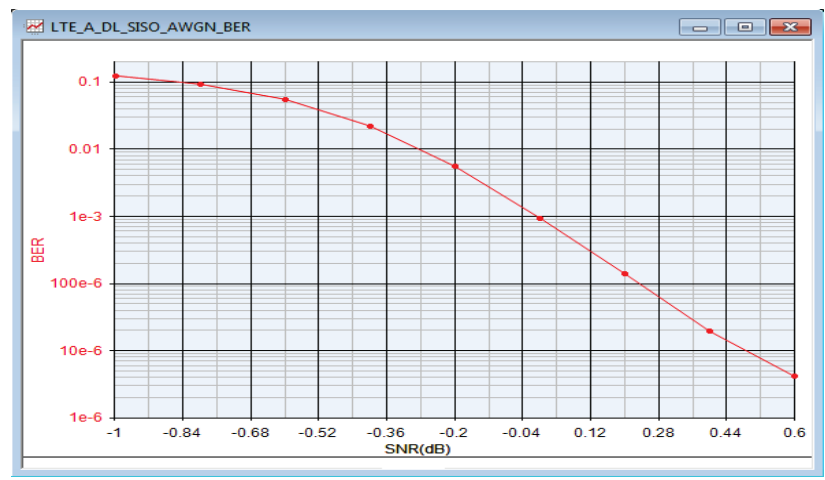

Figure $9 B E R$ vs $S N R$ for the AWGN channel, $M C S / C Q I=6$

This is even more obvious when we eliminate the $S N R$ variable between Fig. 8 and Fig. 9, to graph $B L E R$ vs $B E R$, for the case of $M C S / C Q I=6$ and $g_{\mathrm{RV} 3 / 0}=7 \mathrm{~dB}$ [11], Fig. 10 . 


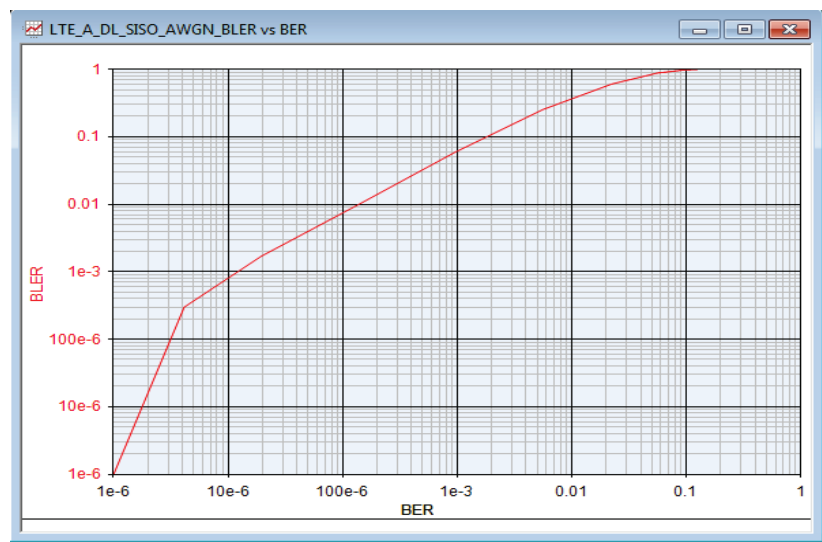

Figure 10 Simulator screen shot of $B L E R_{\mathrm{RES}}$ vs $B E R_{\mathrm{RV} 0}$ for the $A W G N$ channel, $M C S / C Q I=6$

As it is obvious that the curve from Fig. 10 closely matches the corresponding one (for $g_{\mathrm{RV} 3 / 0}=7 \mathrm{~dB}$ ) in Fig. 6 , the residual $B L E R$ model is verified at this level, too.

\section{CONCLUSION}

We developed a novel prediction model for the LTE physical and MAC layer peak transmission performance the irreducible $B L E R$ that determines the HARQ residual channel available to higher-layer protocols.

In this regard, firstly, general pre-HARQ $B L E R$ is estimated for RV0 codeword transmission, as a function of $B E R$, assuming that the cyclic prefix is the efficient enough protection mechanism against inter-symbol interference and long error bursts caused by time dispersion, so that only sporadic and random bit error occurrences might be retained, with moderate mutual interdependence. This implies that it is appropriate to model erroneous bit occurrences within a data block as a sample without replacement, which is consequently described by the hyper geometric statistical distribution, rather than the common binomial one.

Having modelled the pre-HARQ physical layer blockoriented transmission performance, the general residual BLER prediction is extended upwards the protocol stack to take into account the IR-HARQ block-error recovery managed by MAC layer, applying the gain of the RV3 with respect to RV0 for particular MCS/CQI index of interest, where we consider all channel impairments to be abstracted by the equivalent AWGN SNR degradation.

The HARQ BLER prediction model is first validated by Monte-Carlo simulations. In addition, specifically for the post-HARQ residual $B L E R$ model verification in LTE FDD downlink channel conditions, we used a sophisticated industry-standard LTE system-level simulator. The according test results obtained applying both methods exhibit excellent matching with each other, as well as with the residual $B L E R$ prediction.

\section{REFERENCES}

[1] Lipovac, V. (2017). Practical Consistence between Bit Error and Block - Error Performance Metrics up to Application Layer. Wireless Personal Communication, 93(3), 779-793. https://doi.org/10.1007/s11277-014-2228-7

[2] Hamza, M., Lipovac, V., \& Lipovac, A. (2015). BER-based BLER prediction for LTE FDD DL channel with small delay dispersion. Proceedings of the International Wireless
Communications and Mobile Computing Conference (IWCMC), Dubrovnik, 1156-1161. https://doi.org/10.1109/IWCMC.2015.7289246

[3] Lipovac, V. \& Modlic, B. (2016). Modeling OFDM Irreducible BER with Impact of $\mathrm{CP}$ Length and $\mathrm{CFO}$ in Multipath Channel with Small Delay Dispersion. Wireless Communications and Mobile Computing, 16(9), 1065-1077. https://doi.org/10.1002/wcm.2586

[4] Lipovac, A. (2014). OFDM Error Floor Prediction in a Small-Time-Dispersion Channel. Wireless Personal Communications, 75(1), 645-663. https://doi.org/10.1007/s11277-013-1383-6

[5] Wang, X. \& Patzold, M. (2004). A new deterministic process based generative model for characterizing bursty error sequences. 2004 IEEE $15^{\text {th }}$ International Symposium on Personal, Indoor and Mobile Radio Communications (IEEE Cat. No. 04TH8754), Barcelona, Vol. 3, 2134-2139. https://doi.org/10.1109/PIMRC.2004.1368375

[6] $3^{\text {rd }}$ Generation Partnership Project. (2013).Technical Specification Group Radio Access Network, Evolved Universal Terrestrial Radio Access (E-UTRA); User Equipment (UE) radio transmission and reception

[7] Rumnay, M. (2013). LTE and the Evolution of 4G Wireless; Design and Measurements Challenges. $2^{\text {nd }}$ edition, John Wiley \& Sons. https://doi.org/10.1002/9781118799475

[8] Molisch, A. (2011). Wireless Communications (2 ${ }^{\text {nd }}$ ed.). New York, Wiley

[9] Polyanin, A. D. \& Manzhirov, A.V. (1998). Handbook of Integral Equations, CRC Press, Boca Raton https://doi.org/10.1201/9781420050066

[10] Pagès, A. S. (2015). Link Level Performance Evaluation and Link Abstraction for LTE/LTE-Advanced Downlink (Thesis or Dissertation style). PhD dissertation, Department of Signal Theory and Communications, Universitat Politècnica de Catalunya, Barcelona

[11] Ikuno, J. C., Mehlfuhrer, C., \& Rupp, M. (2011). A Novel Link Error Prediction Model for OFDM Systems with HARQ. Proceedings of the IEEE International Conf. on Communications (ICC), Kyoto, 1-5. https://doi.org/10.1109/icc.2011.5962464

[12] Latif, F., Kaltenberger, R. K., \& Olmos, J. (2012). Low Complexity Link Abstraction for Retransmission in LTE/LTE-Advanced with IR-HARQ, in EURO-COST, IC1004 TD(12)0506, Bristol, UK

[13] Agilent Technologies. (2013). SystemVue-electronic system-level design software, technical overview

\section{Contact information:}

Mirza HAMZA, Mr. Sc

Faculty of Electrical Engineering,

University of Sarajevo,

Zmaja od Bosne bb, Kampus Univerziteta,

71000 Sarajevo, Bosnia and Hercegovina

E-mail: mhamza@etf.unsa.ba

Adriana LIPOVAC, PhD

(Corresponding author)

Dept. of Electrical Engineering and Computing,

University of Dubrovnik,

Ćira Carića 4, 20000 Dubrovnik, Croatia

E-mail: adriana.lipovac@unidu.hr

\section{Vlatko LIPOVAC, PhD}

Dept. of Electrical Engineering and Computing,

University of Dubrovnik,

Ćira Carića 4, 20000 Dubrovnik, Croatia

E-mail: vlatko.lipovac@unidu.hr 